\title{
Hemorragia digestiva alta por Sarcoma de Kaposi en paciente con VIH-SIDA
} Upper gastrointestinal bleeding due to Kaposi's Sarcoma in HIV-AIDS patient

\author{
Ana Carolina Freitas Ferreira' ${ }^{1}$, Vanessa Barcelos ${ }^{1}$, Ana Raquel Garrote $^{2}$, Alexandra Caeiro² \\ ${ }^{1}$ Medicina Interna, Hospital do Divino Espirito Santo, Portugal. ${ }^{2}$ Doenças Infecciosas, Hospital de Curry Cabral, Portugal
}

\section{ABSTRACT}

Gastrointestinal bleeding related to Kaposi's Sarcoma is rare in AIDS patients; the etiology of anemia is usually multifactorial.

We describe the case of a HIV infected, 53 year old patient with refractory anemia requiring frequent transfusion support. He was a patient with multiple complications and recent introduction of drugs that could justify myelosuppression and anemia. Due to inconclusive endoscopic examinations, the appearance of melena was the clue of the investigation, which concluded in the diagnosis of duodenal Kaposi's Sarcoma.

Keywords: Anemia; Sarcoma de Kaposi; VIH-SIDA; Hemorragia digestive alta.

Palabras clave: Anemia; Kaposi's sarcoma; HIV-AIDS; Upper gastrointestinal bleeding.

\section{CASE DESCRIPTION}

We present a case of a 53-year-old man, admitted in Emergency Department (ED) for a one-week history of dyspnea and non-productive cough. He had an history of Parkinson's disease and recent diagnosis of HIV-1 infection (subtype B) and syphilis. He reported unprotected heterosexual relationships with several partners.

After evaluation at ED he was hospitalized due to new onset normochromic normocytic anemia (hemoglobin: $7.8 \mathrm{~g} /$ $\mathrm{dl}$ ), acute kidney injury (serum creatinine of $1.27 \mathrm{mg} / \mathrm{dL}$ and urea of $73 \mathrm{mg} / \mathrm{dL}$ ) and respiratory failure, assuming probable opportunistic pulmonary infection. Upon admission, immunovirological staging was performed: CD4 + T cell count of $124 / \mu \mathrm{L}$ (21\%), HIV-1 viral load of 368,000 copies/mL and HLA-B5701 negative. The diagnosis of pneumonia due to parainfluenza virus 1 and 3 was made and non-invasive ventilatory support was provide. During hospitalization he presented with multiple infectious complications.

Introduction of ART with lamivudine, abacavir and dolutegravir was only possible after clinical stabilization and opportunistic infection were excluded.

Meanwhile, the main active problem was persistent pancytopenia (with minimum hemoglobin values of $5.8 \mathrm{~g} / \mathrm{dL}$ ) requiring transfusion support. The laboratorial study was notable for ferropenia $(29 \mathrm{mg} / \mathrm{dL}$ ) and oligoclonal peak gamma protein electrophoresis, but with negative urine or serum immunofixation. There was no identification of lytic or blast lesion on the X-ray of the skull and axial skeleton. Abdominal-pelvic CT scan showed hepatosplenomegaly, ganglia without intra-abdominal pathological volumetric criteria. In bone biopsy, myelogram with moderate plasmacytosis and sterile myelculture were observed. These findings supported the diagnostic impression of a hyperreactive bone marrow in the context of HIV infection, rather than the hypothesis of lymphoproliferative disease.

In the meantime he presented melena. An upper digestive endoscopy was performed showing compatible petechial le-
Fig. 1 (panel A and panel B) - Videoenteroscopy: Hyperemic macules, some with an eroded center and hypertrophic-looking edges extending from the distal duodenum to the ileon. Lesions are very numerous up to the proximal / middle jejunum, decreasing in frequency distally, being scarce in the ileon.
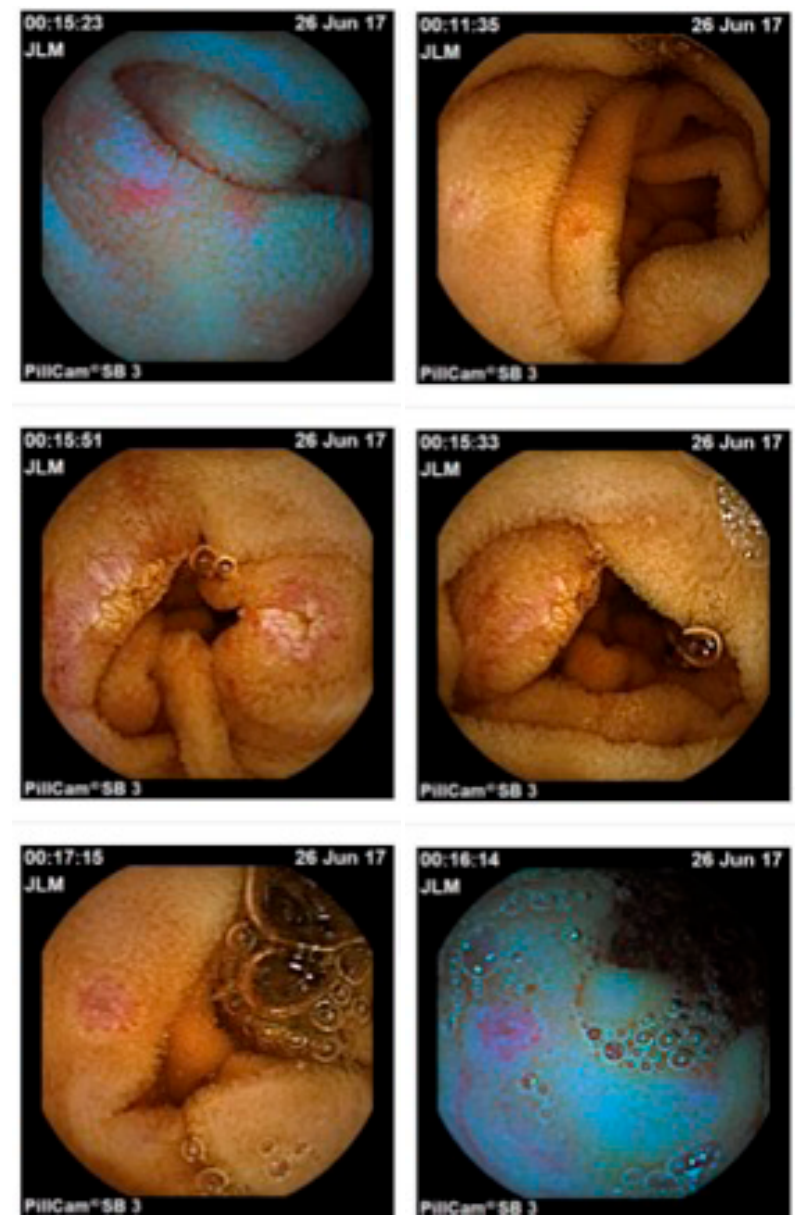

sions resulting from pancytopenia. Total colonoscopy identified sigmoid polyp, histology of tubular adenoma with low-grade dysplasia. In capsule enteroscopy we observed from the distal duodenum, hyperemic macules, some with an eroded center and hypertrophic-looking edges, numerous up to the proximal jejunum, progressively decreasing in frequency distally and 
Fig. 2 (panel A and panel B) - Upper Digestive Endoscopy: Hyperemic macules located in the duodenum, with variable dimensions (3 to $8 \mathrm{~mm}$ ), some eroded and with hypertrophic edges.
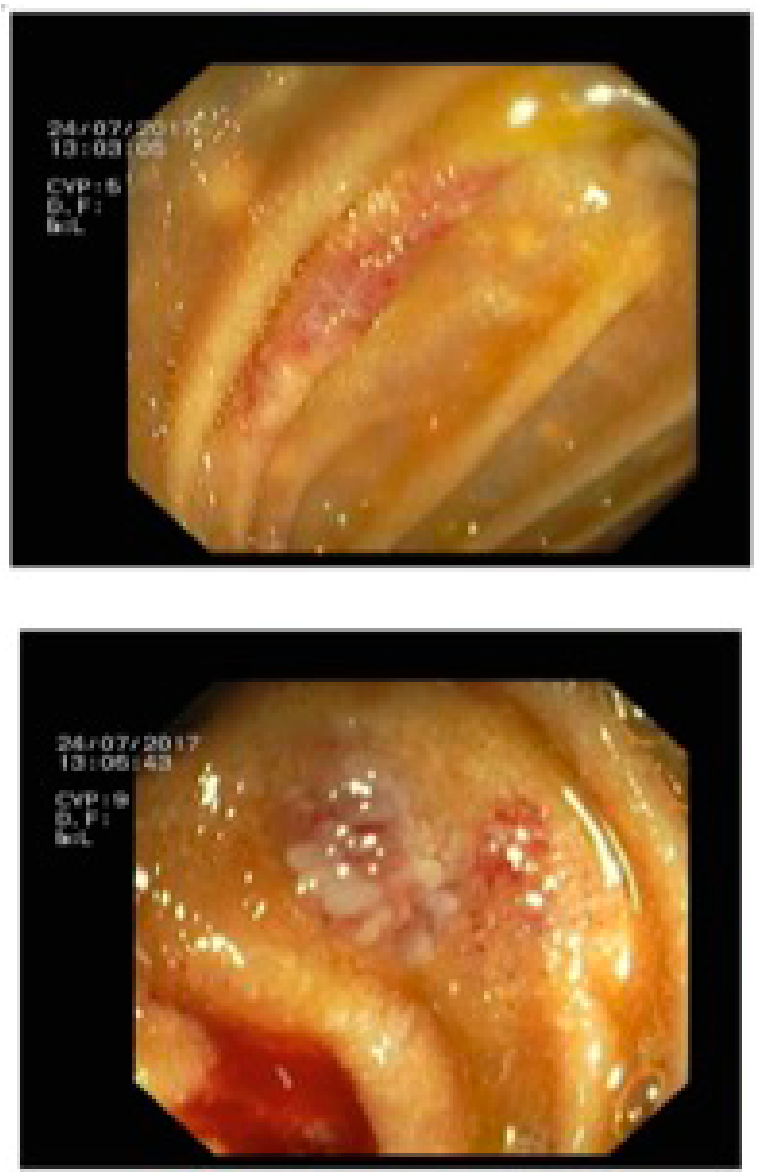

being scarce in the ileon (Fig. 1). Then, double balloon enteroscopy progressing to the distal duodenum allowed us to obtain biopsies (Fig. 2). Macroscopically it consisted of duodenal mucosa fragments with epithelial regenerative aspects, identifying at the level of the chorion several tumoral foci consisting of spindle cell aggregates with KS. Immunostains for human herpesvirus 8 (HHV-8) and CD31 were positive, supporting KS diagnosis. There was no evidence of skin disease attributable to KS. Already on ART and with the diagnosis of visceral KS, the patient was referred to Oncology and treatment was initiated with liposomal Doxorubicin.

At the three-month follow up, the patient showed clinical improvement, reaching a stable hemoglobin value without more transfusions. We opted for clinical surveillance, keeping the ART therapy and the Oncologist follow up.

\section{DISCUSSION}

Upper gastrointestinal bleeding (UGB) is a common entity with an estimated annual incidence of 40 to 150 cases per 100,000 individuals ${ }^{1}$. KS is an extremely rare cause of UGB². Despite decades of research, its etiopathogenesis remains unclear and is found in association with the HHV-8 infection $^{2-6}$. Clinically, it is manifested by nodular vascular lesions that affect the skin surface, mucous membranes and/or viscera ${ }^{5}$. Visceral involvement is common in AIDS-related KS but is often asymptomatic. The clinical data regarding the visceral presentation of $\mathrm{KS}$ is limited to case reports and series, mostly with concomitant skin lesions ${ }^{3}$. In our literature review there was no documented Gastrointestinal SK presentation with merely duodenum involvement.

Gastrointestinal SK (GISK) diagnosis depends on endoscopic examinations, however the findings may be nonspecific and biopsies should be obtained. The presence of HHV-8 is required and immunohistochemical testing is recommended for all lesions with spindle cell morphology. The therapeutic approach depends on the KS variant and is individualized to the patient's profile and the extent of the disease. Among patients with visceral involvement, the combination of ART with systemic chemotherapy showed favorable results, with reduced associated morbidity and mortality rates. Liposomal doxorubicin is the chemotherapeutic agent of choice ${ }^{5}$.

Some specialists support the use of endoscopic examinations in selected patients, with the main objectives of early detection and institution of survival improvement therapy. Recent retrospective studies suggest that low CD4 + T lymphocyte counts (less than 100 cells / $\mu \mathrm{L}$ ), men who have sex with men, and the presence of cutaneous KS are predictive factors for GIKS $2-7,9$.

We highlight a case of a heterosexual man presenting with symptomatic anemia and without the clue of typical skin lesion. Persistent investigation enabled us to report this unusual localization of gastrointestinal SK. Therefore, it is important to maintain a broad differential diagnosis for anemia in HIV-infected individuals and to remember that atypical presentations are possible.

\section{REFERENCES}

1. Jiang $M, C$ Chen $P, G a o Q$. Systematic review and net-work meta-analysis of upper gastrointestinal hemorrhage interventions. Cell Physiol Biochem. 2016; 39: 2477 91.

2. Ling J, Coron R, Basak P, Jesmajian S. Recurrent lower gastrointestinal bleeding duo to primary colonic Kaposi's sarcoma in a patient with AIDS. International $\mathrm{J}$ of STD \& AIDS. 2013; 24 (11): 908-11.

3. Mansfield S, Stawicki S, Forbes R, et al. Acute upper gastrointestinal bleeding secondary to Kaposi Sarcoma as initial presentation of HIV infection. J Gastrointestin Liver Dis. 2013; 22 (4): 441-5.

4. Rezende R, Kahwage RL, Vieira da Costa T, et al. Upper gastrointestinal Kaposi's sarcoma in HIV-infected patients: ten years of endoscopy observation at a single Brazilian center. Inter J Infect Dis. 2015; 39: 110-5

5. Lee A, Brenner L, Mourad B, et al. Gastrointestinal Kaposi's sarcoma: Case report and review of the literature. World J Gastrointest Pharmacol Ther. 2015; 6 (3): 8995.

6. Lin CH, Hsu CW, Chiang YJ, et al. Esophageal and gastric Kaposi's sarcomas presenting as upper gastrointestinal bleeding. Chang Gung Med J. 2002; 25 (5): 329-33.

7. Parent $F$, Cernushi M, Orlando $G$ et al. Kaposi's sarcoma and AIDS: frequency of gastrointestinal involvement and its effect on survival. A prospective study in a heterogeneous population. Scand J Gastroenterol. 1991; 26: 1007-12.

8. Berg K, Murphy EL, Pretorius L, Louw VJ. The impact of HIV-associated anaemia on the incidence of red blood cell transfusion: Implications for blood services in HIV-endemic contries. Transfus Apher Sci. 2015; 51 (3): 10-8.

9. Nagata $\mathrm{N}$, Shimbo T, Yazaki $\mathrm{H}$, et al. Predictive clinical factors in the diagnosis of gastrointestinal Kaposi's Sarcoma and its endoscopic severity. PLOS One. 2012; 7: e46967. 